\title{
Turbulent broadening of optical spectra in ultralong Raman fiber lasers
}

\author{
S. A. Babin, ${ }^{1, *}$ V. Karalekas, ${ }^{2, \dagger}$ E. V. Podivilov, ${ }^{1}$ V. K. Mezentsev, ${ }^{2}$ P. Harper, ${ }^{2}$ J. D. Ania-Castañón, ${ }^{2,3}$ and S. K. Turitsyn ${ }^{2}$ \\ ${ }^{1}$ Institute of Automation and Electrometry, SB RAS, Novosibirsk 630090, Russia \\ ${ }^{2}$ Photonics Research Group, Aston University, Birmingham, B4 7ET, United Kingdom \\ ${ }^{3}$ Instituto de Óptica “Daza de Valdés,” CSIC, Serrano 121,28006 Madrid, Spain
}

(Received 2 July 2007; published 3 March 2008)

\begin{abstract}
We study the properties of radiation generated in ultralong fiber lasers and find an interesting link between these optical systems and the theory of weak wave turbulence. Experimental observations strongly suggest that turbulentlike weak interactions between the multitude of laser cavity modes are responsible for practical characteristics of ultralong fiber lasers such as spectra of the output radiation.
\end{abstract}

DOI: 10.1103/PhysRevA.77.033803

PACS number(s): 42.55.Wd, 42.55.Ah, 42.65.Sf

\section{INTRODUCTION}

Wave turbulence is a fundamental nonlinear phenomenon that occurs in a variety of nonlinear dispersive physical systems (see, e.g. [1] and references therein). In many wavebearing systems nonlinear effects are relatively small and interaction between waves is weak. Weak wave turbulence theory deals with the statistical behavior of a large number of weakly interacting waves with random phases. There are two primary types of physical systems with turbulentlike behavior. The first case, mostly associated to the term turbulence (or developed turbulence), occurs when the scales at which waves are excited and disappeared are well separated and the turbulent energy transfer between spectral components in the inertial interval does not depend much on the details of system specifics at the edges of the spectrum [1]. In the second case, the scales cannot be fully separated and turbulentlike behavior does not have a well-defined inertial interval. In this paper we present what seems to be an example of weak wave turbulence behavior of the second type, as exhibited in ultralong Raman fiber lasers. Moreover, we provide evidence strongly suggesting that turbulentlike weak interactions between a very large number of cavity modes are responsible for practical characteristics of ultralong Raman fiber lasers (RFLs) such as spectral broadening of the generated radiation.

RFLs rely on stimulated Raman scattering (SRS), which shifts the spectrum of the propagating electromagnetic radiation toward longer wavelengths. In contrast with bulk media, optical fibers, with their low light absorption ( $\alpha$ $\sim 0.2 \mathrm{~dB} / \mathrm{km}$ at $\lambda \sim 1.55 \mu \mathrm{m})$ and small core diameters $(5-10 \mu \mathrm{m})$ that allow high-intensity light propagation over long distances, provide a much stronger SRS effect. The high-intensity wave acts as a pump, inducing Raman amplification of the redshifted Stokes wave with gain coefficient $\sim 1 \mathrm{~dB} \mathrm{~km}^{-1} \mathrm{~W}^{-1}$, typically sufficient to overcome fiber attenuation at powers $\sim 1 \mathrm{~W}$. As a result, an integral positive Raman gain over a given fiber length is enough to achieve lasing if a cavity is formed by adding fiber Bragg gratings at the fiber ends, resonantly reflecting the forward and back-

\footnotetext{
*babin@iae.nsk.su

†karalekv@aston.ac.uk
}

ward propagating Stokes [2] waves. RFLs have become very popular, presenting important applications in optical communications, where they are used both as signal and pump sources in distributed Raman amplified systems (see [3] and references therein).

Recently, a quasilossless signal transmission scheme was proposed and implemented, based upon the concept of ultralong $(75 \mathrm{~km}$ ) Raman laser architecture (see, e.g. [4] and references therein). In such a system, the combined forwardand backward-propagating Stokes wave generated at $\sim 1455 \mathrm{~nm}$ inside the high- $Q$ RFL cavity (formed by the transmission fiber itself) experiences reduced variations along the fiber span. Hence the generated intracavity power can be used as a homogeneous, stable secondary pump to provide a nearly constant (along the fiber) Raman gain for an optical signal transmitted within a bandwidth of $36 \mathrm{~nm}$ centered about $1550 \mathrm{~nm}$. By adjusting the total gain to equal optical loss, quasilossless transmission can be implemented [4]. The substantial increase of the RFL cavity length leads to a new interesting class of lasers with potentially different physical mechanisms underlying their operation. In this context, various fundamental questions arise: What are the limits of the cavity length for laser operation? What are the particular spectral features of ultralong RFLs-cavity mode structure, output spectrum, corresponding coherence propertiesand temporal properties of the radiation generated inside the cavity?

We address these questions through the analysis of the spectral and temporal characteristics of ultralong RFLs with cavity lengths from $6.6 \mathrm{~km}$ to $84 \mathrm{~km}$ (incidentally, the current record cavity length, to our knowledge). We will show that important characteristics of such ultralong fiber lasers are directly explainable by the occurrence of weak turbulentlike interactions of a huge number (up to 100 millions) of longitudinal laser modes.

\section{EXPERIMENT}

The basic design of an ultralong RFL is depicted in Fig. 1. Two equal-power depolarized pumps centered at $1365 \mathrm{~nm}$ and coupled into each end of a standard single mode fiber (SMF) span are used. Two highly reflective $(\sim 98 \%)$ fiber 


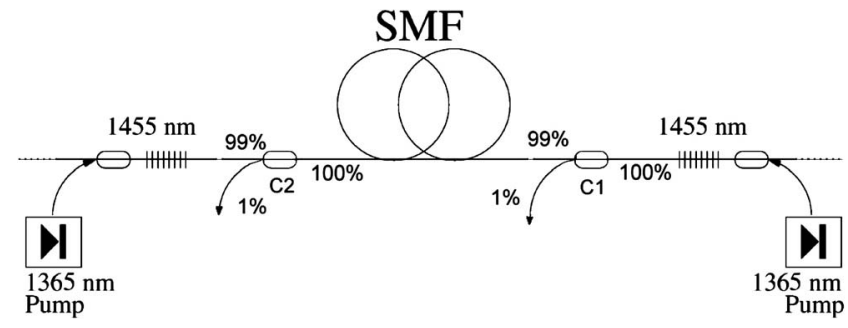

FIG. 1. Schematics of the ultralong Raman fiber laser.

Bragg gratings (FBG) with a bandwidth of $\sim 1 \mathrm{~nm}$, centered at $1455 \mathrm{~nm}$, delimit the fiber span forming a high- $Q$ cavity that traps the first Stokes wave. When the pump power reaches threshold, the RFL starts lasing at $1455 \mathrm{~nm}$. Span lengths of $6.6,22,44$, and $84 \mathrm{~km}$ were tested, in order to study RFL properties dependence on cavity length. Two 99:1 couplers were placed near the FBGs at the right $(C 1)$ and left $(C 2)$ ends of the span to monitor the generated intracavity Stokes wave power and its optical and radio frequency (RF) spectra. The RF spectrum, monitored using a photodetector and an electrical spectrum analyzer, displays intermode beating peaks with a clear mode structure despite the large cavity length up to some critical power (see [5] for details). The optical spectrum of the generated Stokes wave was monitored through an optical spectrum analyzer with resolution of $\sim 0.01 \mathrm{~nm}$. The Stokes wave power was measured by a power meter, whereas its temporal behavior was studied with a fast oscilloscope with a 50 ps resolution.

Figure 2(a) shows the total intracavity power of the Stokes wave $(1455 \mathrm{~nm})$, measured via the $1 \%$ port at point $C 2$, as a function of the total pump power at $1365 \mathrm{~nm}$. The generated power exhibits typical laser behavior. Above the threshold required for the SRS to overcome fiber attenuation ( $\left.\alpha_{1455} \approx 0.25 \mathrm{~dB} / \mathrm{km}\right)$ and lumped losses of FBGs, couplers, and connections $\left(\delta_{0} \approx 1.4 \mathrm{~dB}\right)$, the laser starts generation at $1455 \mathrm{~nm}$. Experimental results are in good agreement with modeling [solid lines in Fig. 2(a)] of the modes dynamics using ordinary differential equations describing four-wave mixing (FWM) interactions. Though the equations are dynamic, the solutions should be treated as stochastic: The FWM nonlinear process engages a huge number of rapidly oscillating terms with different amplitudes and phases.
Above threshold, the Stokes grows nearly linearly with increasing pump power. As expected, the longer the cavity, the lower the generated $1455 \mathrm{~nm}$ power, due to higher cavity losses caused by the attenuation of the pump $\left(\alpha_{1365}\right.$ $\approx 0.31 \mathrm{~dB} / \mathrm{km})$ and Stokes waves, which raise the threshold. Our experimental threshold values are in complete agreement with the simple theoretical RFL model, which was already confirmed by experiments with short fiber spans [6]. For longer spans, distributed losses remain the main factor and the threshold is nearly proportional to the length. Despite this, even for the longest cavity length of $84 \mathrm{~km}$, the threshold pump power is rather moderate, $\sim 0.7 \mathrm{~W}$.

The evolution of the intracavity Stokes wave optical spectra is shown in Fig. 2(b) for the $6.6 \mathrm{~km}$ case. The spectrum is rather narrow near threshold and broadens with increasing pump power. Furthermore, the broadened spectrum acquires clear exponential tails for all cavity lengths studied. To trace the spectral structure dependence on the boundary condition, we used apodized FBGs with ripples in the short-wave wing of their reflection profile. Figure 2(b) shows that the spectrum for Stokes power $=265 \mathrm{~mW}$ has the same low wavelength ripples as the FBGs, which are not seen for Stokes power $=714 \mathrm{~mW}$ : The generated spectra follow the FBGs reflection profile at low powers, but are not influenced by the boundary conditions at higher powers. A drift towards longer wavelengths with increasing power was observed, but measurements of the gratings spectral response revealed a shift of the central wavelength of the FBGs - attributed to thermal expansion-as the cause. The spectral width of the Stokes wave increases nonlinearly with power, as seen in Fig. 3(a), and does not vary significantly with length for identical powers. A square-root fit appears to be a good approximation of the spectral width dependence on the power at $-3 \mathrm{~dB}$.

The broadening mechanism and its dependence on input power can be understood from the analysis of the interaction between the intracavity longitudinal modes. In a laser cavity, the spacing between neighboring modes, see, e.g. [7], is given by $\Delta=c / 2 L$, where $c$ is the speed of light in the fiber and $L$ is the cavity length. Therefore, an increased cavity length leads to a reduced mode spacing $\Delta$, which manifests itself in the RF spectrum as the distance between intermode beating peaks, whereas the width of the peaks is determined by the relative dephasing of the modes [5]. For $L>6 \mathrm{~km}$, (a)

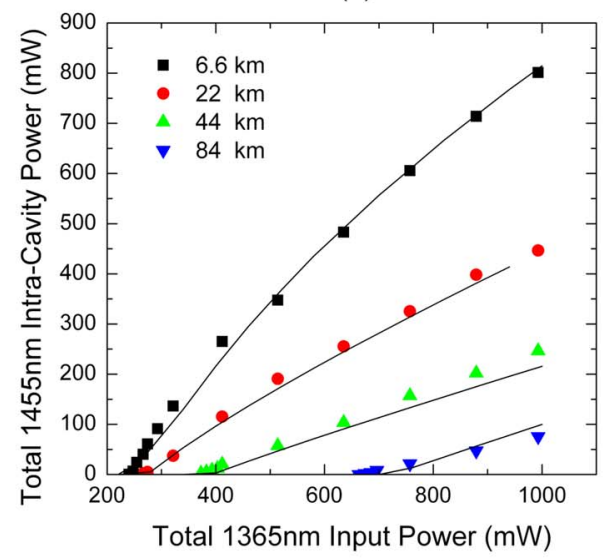

(b)

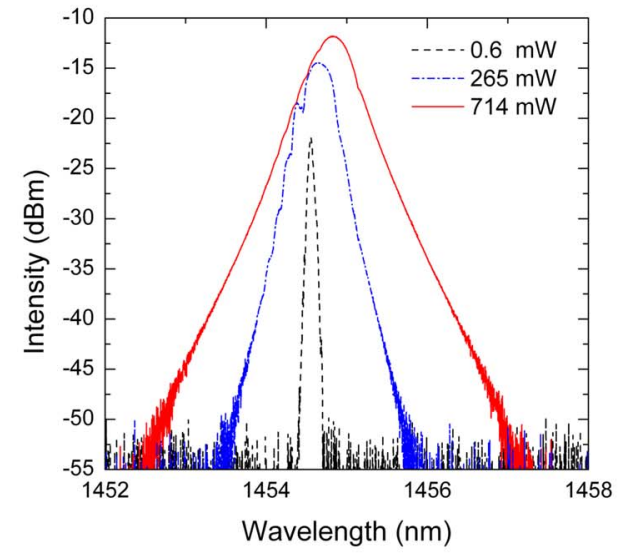

FIG. 2. (Color online) (a) Total intracavity power at $1455 \mathrm{~nm}$ as a function of the total pump power at $1365 \mathrm{~nm}$ : Experimental points and numerical simulation (solid curves). (b) $1455 \mathrm{~nm}$ spectrum measured at point $C 2$; Stokes power $=0.6,265$, and $714 \mathrm{~mW}$ for $L=6.6 \mathrm{~km}$. 
(a)

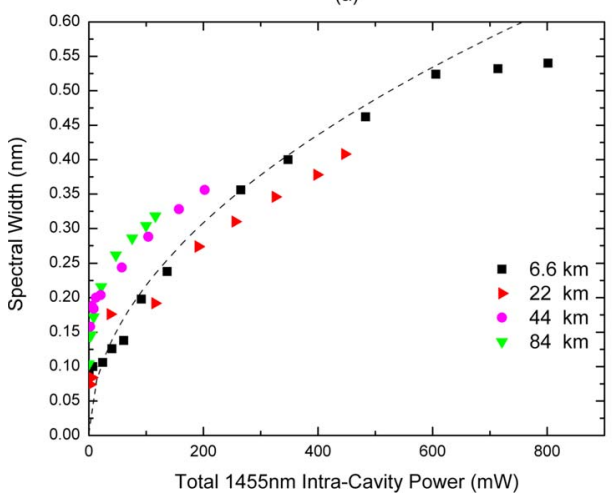

(b)

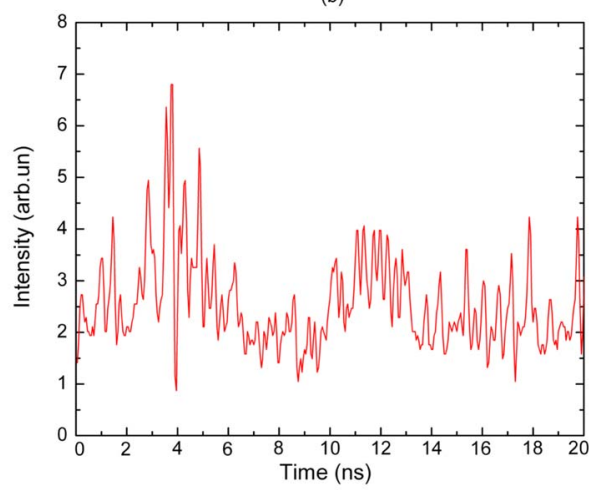

FIG. 3. (Color online) $1455 \mathrm{~nm}$ spectral widths at $3 \mathrm{~dB}$ (a) as a function of $1455 \mathrm{~nm}$ power for different fiber cavity lengths $L=6.6,22,42$, and $84 \mathrm{~km}$. The dashed lines represent the fit by function $y=A x^{1 / 2}$. (b) Typical temporal behavior of $1455 \mathrm{~nm}$ intensity, $L=22 \mathrm{~km}, \quad$ and $I$ $\sim 100 \mathrm{~mW}$.
$\Delta<20 \mathrm{kHz}$ and the number of modes in a typical bandwidth of $\sim 200 \mathrm{GHz}(\sim 2 \mathrm{~nm})$ can be estimated as $N>10^{7}$. Nonlinear interaction of the modes through multiple FWM processes results in the stochastic evolution of the amplitudes and phases of the individual modes $[8,9]$.

We would like to stress that such a stochastic, turbulentlike behavior of the modes leads to a rather specific broadening of the RF spectra, proportional to the generated intensity. The width of the intermode beating peaks $D$ appears almost independent of the cavity length and grows linearly with increasing Stokes wave intensity $I$ [see, Fig. 4(a)], confirming the major role of nonlinear attenuation and the stochastic nature of the cavity mode interactions. At the same time, $\Delta$ decreases with cavity length from $\Delta \sim 15.5 \mathrm{kHz}$ at $L=6.6 \mathrm{~km}$ to $\Delta \sim 1.2 \mathrm{kHz}$ at $L=84 \mathrm{~km}$. Corresponding spacing values are shown in Fig. 4(a) by horizontal lines. The power limit for a resolvable mode structure may be defined as the value for which the modal width equals the mode spacing (i.e., $D \approx \Delta$ ) corresponding to the intersection of the linear fit in for the modal width in Fig. 4(a) (solid line) with the horizontal lines for the mode spacing of each specific length. Thus the defined maximum power with a resolved mode structure is inversely proportional to the length: $I_{\max }$ $\propto L^{-1}$, see Fig. 4(b) and is as low as $\sim 100 \mathrm{~mW}$ for the $84 \mathrm{~km}$ cavity. Higher powers result in the generation of "modeless" stochastic spectra with an exponential-wing envelope. Simultaneously, in the time domain, we observe stochastic behav-

ior with fluctuations in various time scales with amplitude of noisy spikes reaching $>50 \%$ of average level; see Fig. 3(b) (compare with $[10,11]$ ).

\section{RESULTS AND DISCUSSION}

The theoretical analysis of the spectrum formation requires the use of statistical techniques [1] instead of the dynamical formalism commonly used in fiber optics; see, e.g. [12]. The first attempt in this direction was made in [8,9] for RFL with $L<1 \mathrm{~km}$, where the wave kinetic equation for spectral density evolution was derived, using a technique of averaging and splitting of correlation functions in the context of the weak turbulence theory [1]. For a longitudinally homogeneous Stokes wave intensity (proven in shorter RFL, see [6]) and the typical Gaussian shaped reflection spectrum of FBGs, the analytical solution for the Stokes spectrum inside the cavity takes a hyperbolic secant shape with exponential tails [8]. Moreover, the spectral width grows with increasing power according to a square-root law. Experimental study of $L \sim 0.37 \mathrm{~km}$ long RFL spectra in the region of normal dispersion $(\lambda \sim 1.23 \mu \mathrm{m})$ has demonstrated very good quantitative agreement with analytics both in shape and broadening [9].

(b)

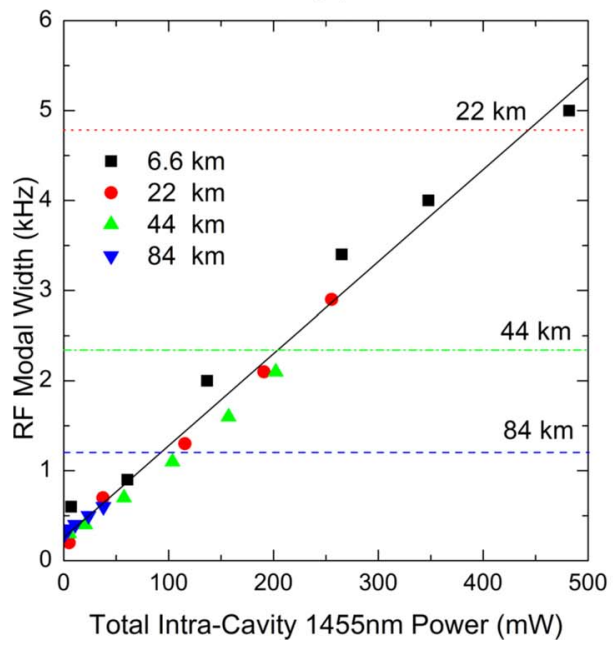

FIG. 4. (Color online) (a) Experimental values for spectral width of the RF peaks [5] as a function of the total intracavity power at $1455 \mathrm{~nm}$ for cavity lengths of $6.6,22,44$, and $84 \mathrm{~km}$ and corresponding mode spacing values marked by dots $(22 \mathrm{~km})$, dash dots $(44 \mathrm{~km})$, and dashes $(84 \mathrm{~km}) ; 15.5 \mathrm{kHz}$ spacing at $6.6 \mathrm{~km}$ lies beyond the graph. (b) Maximum power with resolved mode structure as a function of the cavity length; solid lines are (a) linear fit and (b) $A / x$ fit. 
Extending the laser cavity to $L \sim 80 \mathrm{~km}$ greatly increases the number of modes $\left(\sim 10^{8}\right)$, enhancing the effects of wave turbulence. This is confirmed by the strong broadening of the Stokes spectra, which is significant even at $\mathrm{mW}$ level (see Figs. 2 and 3). The spectrum has well-defined exponential tails. Moreover, the dependence of the $-3 \mathrm{~dB}$ width with the intracavity power [see Fig. 3(a)] is nonlinear (close to square root) as predicted analytically [9]. Note, however, that the analytical theory is not directly applicable in the case of ultralong Raman fiber lasers. The main issues for a quantitative analysis which are not resolved by the simple theory are (1) the reflection profiles of the FBGs used in the laser cavity are not purely Gaussian, (2) longitudinal homogeneity is not an adequate assumption for such a long cavity, and (3) the Stokes wavelength corresponds to anomalous dispersion, and not normal dispersion as in $[8,9]$.

The analysis of the impact of the FBG reflection profile demonstrates the washing out of the FBG-induced ripples after propagation through the fiber at high powers. Using an additional splitter at intermediate points, we observed that the spectrum acquired its characteristic exponential tails without ripples after $<6 \mathrm{~km}$ propagation. Therefore, we conclude that system memory of the FBG profile is lost in the nonlinear turbulent evolution of the cavity modes.

For the qualitative analysis of the experimental results we apply the analytical approach developed in [9]. Intracavity power $I$ generated at pump power $P$ is derived from the integral equality condition of gain and loss:

$$
2 L \alpha_{I}+\bar{\delta}(I)=2 L g_{R} P_{0} \frac{1-\exp \left(-\alpha_{P} L-g_{R} \frac{\lambda_{I}}{\lambda_{P}} I L\right)}{\alpha_{P} L+g_{R} \frac{\lambda_{I}}{\lambda_{P}} I L} .
$$

The integral gain in the right-hand side is reduced with increasing $I$ because of the pump depletion. Note that variations of intracavity power $I(z)$ along the fiber are assumed to be small. The average nonlinear losses $\bar{\delta}(I)$ in the left-hand side grow with increasing $I$ due to the spectral broadening and the corresponding effective increase of the FBG transmission: $\bar{\delta}(I)=\delta_{0}+\delta_{\mathrm{NL}}(I)$, where $\delta_{0}$ are the effective losses at the central frequency, including lumped losses, and $\delta_{\mathrm{NL}}(I)$ $\propto 2 \gamma I L$ is the attenuation induced by FWM conversion from the center to the side spectral components. Multiple FWM processes being a particular effect of the Kerr nonlinearity lead to relative dephasing of the neighboring modes that manifests itself in a broadening of the intermode beating peaks in RF spectra. Note that other manifestations of the Kerr nonlinearity such as self-phase or cross-phase modulation (SPM, XPM) do not lead to the broadening as they change the mode phases synchronously. Moreover, the SPM/ XPM is a purely dynamic effect and cannot lead to random phase shifts. As a result, the corresponding terms in the wave kinetic equation turn to zero; see Appendix in [9]. Near the threshold, mode competition effects may also have some influence, but with increasing of modes number their role is reduced.
At high power $I$, the width of the mode beating in the RF spectra defined by the multiple FWM processes is: $D$ $=\delta_{\mathrm{NL}} / \tau_{\mathrm{rt}}=K \gamma c I$, where $\tau_{\mathrm{rt}}=2 L / c$ is the round trip time, $\gamma$ $=1.4 \mathrm{~W}^{-1} \mathrm{~km}^{-1}$ is the Kerr nonlinearity coefficient, $c=2$ $\times 10^{5} \mathrm{~km} / \mathrm{s}$ is the speed of light in the fiber, and $K$ is a coefficient depending on the spectrum shape [5], which can be evaluated as $1 / 5$ in the present conditions, since the proportionality coefficient is $K \gamma c=10 \mathrm{kHz} / \mathrm{W}$ [see Fig. 4(a)]. For normal cavity dispersion [9] one can neglect the backward FWM process: conversion from the side components into the central part of the spectrum. However, in the current case of anomalous dispersion the phase correlations between the waves induced by modulation instability have to be taken into account. The distance between correlated components is defined by the modulation instability resonant frequency $\Omega_{\mathrm{MI}} \approx \sqrt{\gamma I / \beta}$, where $\beta=8.9 \mathrm{~nm}^{-2} \mathrm{~km}^{-1}$ is the dispersion coefficient. This effect limits the resulting spectral width by suppressing further broadening, and the value $2 \Omega_{\mathrm{MI}}[\mathrm{nm}]$ $\approx 0.75 \sqrt{I[W]}$ could define the spectral width observed in the experiments. Indeed, the square-root fit of the experimental points [see Fig. 3(a)] is in reasonable agreement with this simple estimate. The "limiting" role of this effect is also confirmed by the spectral evolution after FBG reflection, in which the narrow spectrum broadens with propagation but takes its final form after several kilometres without further changes. Deviations from the square root law are seen to be more significant for higher power and longer cavities, which can be explained by the increasing longitudinal inhomogeneity.

\section{CONCLUSION}

In conclusion, the performed experimental and theoretical study strongly suggests the presence of a new operating regime in ultralong lasers (RFLs) - turbulent generation of a multitude (up to $\sim 10^{8}$ ) of interacting cavity modes. The observed weak wave turbulence effects are of fundamental interest and have also a direct impact on RFL applications in communications, e.g., the performance of quasilossless links based on ultralong Raman lasers [4]. This class of ultralong lasers is characterized by a range of particular properties, such as the exponential wings of the generated optical spectra, which broaden nonlinearly (close to square root) with increasing intensity. Their mode structure is resolvable in the $\mathrm{RF}$ spectra, in a limited power interval from the generation threshold up to a maximum power dependent on cavity length. Above a given power value, the stochastic mode dephasing due to uncorrelated fluctuation of the mode frequencies induced by FWM leads to broadening prevailing over mode separation and to the generation of a "modeless" or "quasicontinuous" spectrum. The corresponding power limit is about $100 \mathrm{~mW}$ for the $84 \mathrm{~km}$ cavity. Assuming that a more-or-less stable measurable intracavity RFL power is around $10 \mathrm{~mW}$, we predict that the maximum cavity length with resolvable mode structure might be increased up to $1000 \mathrm{~km}$. This effect can be used to define the limiting length or operational power of "ultralong" fiber lasers as the point at which, as a result of the weak wave turbulent nonlinear dephasing, the generated light "forgets" about the cavity mode structure. 


\section{ACKNOWLEDGMENTS}

S.A.B. and E.V.P. acknowledge financial support by the integration grant N31 of the Siberian Branch of the Russian Academy of Sciences and the governmental program of sup- port of leading scientific schools. J.D.A.C. and S.K.T acknowledge the financial support of the Engineering and Physical Sciences Research Council and the Royal Society. P.H. acknowledges financial support from The Nuffield Foundation Grant.
[1] V. E. Zakharov, V. S. L'vov, and G. Falkovich, Kolmogorov Spectra of Turbulence I: Wave Turbulence (Springer-Verlag, Berlin, 1992).

[2] S. G. Grubb, T. Strasser, W. Y. Cheung, W. A. Reed, V. Mizrahi, T. Erdogan, P. J. Lemaire, A. M. Vengsarkar, D. J. Digiovanni, D. W. Peckham, and B. H. Rockney, in Optical Amplifiers and Their Applications, OSA Technical Digest Series, 1995 (Optical Society of America, Washington, D.C., 1995), pp. 197-199, paper SaA4.

[3] C. Headley and G. P. Agrawal, Raman Amplification in Fibre Optical Communication Systems (Academic Press, New York, 2004).

[4] J. D. Ania-Castañón, T. J. Ellingham, R. Ibbotson, X. Chen, L. Zhang, and S. K. Turitsyn, Phys. Rev. Lett. 96, 023902 (2006).

[5] S. A. Babin, V. Karalekas, P. Harper, E. V. Podivilov, V. K. Mezentsev, J. D. Ania-Castañón, and S. K. Turitsyn, Opt. Lett.
32, 1135 (2007).

[6] S. A. Babin, D. V. Churkin, and E. V. Podivilov, Opt. Commun. 226, 329 (2003).

[7] O. Svelto, Principles of Lasers, 1st ed. (Springer, New York, 1999).

[8] S. A. Babin, D. V. Churkin, A. E. Ismagulov, S. I. Kablukov, and E. V. Podivilov, Opt. Lett. 31, 3007 (2006).

[9] S. A. Babin, D. V. Churkin, A. E. Ismagulov, S. I. Kablukov, and E. V. Podivilov, J. Opt. Soc. Am. B 24, 1729 (2007).

[10] D. Hochheiser, J. V. Moloney, and J. Lega, Phys. Rev. A 55, R4011 (1997).

[11] F. Mitschke, G. Steinmeyer, and A. Schwache, Physica D 96, 251 (1996).

[12] G. P. Agrawal, Nonlinear Fibre Optics (Academic Press, London, UK, 1995). 